\title{
Increase in Plasma Concentrations of Geranylgeranoic Acid after Turmeric Tablet Intake by Healthy Volunteers
}

\author{
Maiko Mitake ${ }^{1}$, Hiroko Ogawa ${ }^{2}$, Kazuo Uebaba ${ }^{2}$ and Yoshihiro Shidoji ${ }^{1 *}$ \\ ${ }^{1}$ Molecular and Cellular Biology, Graduate School of Human Health Sciences, \\ Siebold University of Nagasaki, Nagayo, Nagasaki 851-2195, Japan \\ ${ }^{2}$ Department of Presymptomatic Health Promotion, Institute of Natural Medicine, \\ University of Toyama, Tomosugi, Toyama 939-8224, Japan
}

Received 11 November, 2009; Accepted 4 January, 2010; Published online 10 April, 2010

\begin{abstract}
Summary Geranylgeranoic acid (GGA) is one of the most potent cancer-preventive acyclic retinoids. GGA has been shown to induce cell death in human hepatoma-derived HuH-7 cells. We have recently reported the natural occurrence of GGA and its related compounds in several medicinal herbs such as turmeric, basil, rosehip, cinnamon and others [Shidoji and Ogawa, J. Lipid Res., 45: 1092-1103, 2004]. In the present study, we performed oral administration of turmeric tablets to healthy volunteers in order to investigate bioavailability of natural GGA. By using liquid chromatography/mass spectrometry, authentic GGA was eluted at a retention time of around $18 \mathrm{~min}$ as a negative ion of $\mathrm{m} / \mathrm{z}, 303.4$. With healthy volunteers, plasma GGA was detected prior to the tablet intake and its concentrations were increased at $2 \mathrm{~h}$ after its intake and maintained at higher level until $4 \mathrm{~h}$, suggesting an efficient bioavailability of preformed GGA in the turmeric tablets through oral administration. These results indicated that GGA in the turmeric tablet was absorbed as an intact form from intestinal mucosa. The present study provides a clue to conduct a research for cancer preventive roles of GGA in a number of spices.
\end{abstract}

Key Words: turmeric tablet, geranylgeranoic acid, intestinal absorption, arachidonic acid

\section{Introduction}

The efficacy of a synthetic 20-carbon polyprenoic acid (alltrans 3,7,11,15-tetramethyl-2,4,6,10,14-hexadecapentaenoic acid) on prevention of second primary hepatoma has been proven in a double-blinded and randomized phase II clinical trial with postoperative hepatoma patients with few side effects [1], and recently, it was revealed that the polyprenoic acid significantly increased a 5-year survival rate after a radical therapy of primary hepatoma in these patients [2]. We have also shown that the polyprenoic acid binds to cellular retinoic acid binding protein (CRABP) [3] as well

*To whom the correspondence should be addressed.

Tel/Fax: +81-95-813-5207

E-mail: shidoji@sun.ac.jp as to nuclear retinoid receptors [4], exerts transcriptional activation of some hepatocyte-specific genes in hepatoma cells [5], and has preventive actions in chemical and spontaneous hepatocarcinogenesis [ 6 ]. In this context, we named this compound "acyclic retinoid" [7].

Acyclic retinoid induced cell death in human hepatomaderived cell lines, HuH-7 and PLC/PRF5, although natural retinoids such as retinoic acid failed to induce cell death at least at the same condition [8]. Therefore, we have speculated that acyclic retinoid may have different metabolism and signalling from retinoic acid in cells and animals, and may mimic other biological components such as acyclic diterpenoids, for example, geranylgeranoic acid (GGA), from which acyclic retinoid, 4,5-dehydroGGA, differs for the additional double bond at a position between $\mathrm{C} 4$ and $\mathrm{C} 5$. GGA has been considered as a synthetic compound for a long time, but its chemical structure suggests that GGA may 
occur as a natural substance in a group of acyclic diterpenoids. Recently, we reported the natural occurrence of GGA in several medicinal herbs (schisandra, licorice, etc.) used in traditional medicine, Kampo and Ayurveda, as well as ordinary herbs (turmeric, basil, rosehip, cinnamon, etc.) [9].

In the present study, we examined a bioavailability of GGA in commercially available turmeric tablets by oral administration of the tablet to healthy volunteers and liquid chromatography/mass spectrometry (LC/MS) analysis of plasma GGA.

\section{Materials and Methods}

\section{Chemicals}

All-trans GGA was obtained from Kuraray (Kurashiki, Okayama, Japan). n-Hexane, dehydrated diethyl ether, 2 M sodium hydroxide solution, and $2 \mathrm{M}$ hydrochloric acid were from Wako Pure Chemical Industries (Osaka, Japan). Nmethyl-N-nitro-N-nitrosoguanidine (MNNG) was purchased from GL Science Inc. (Tokyo, Japan). Arachidonic acid (ARA) was from Sigma-Aldrich (St Louis, MO). Methanol, methanol for HPLC, and ethanol were from Kanto Chemical Co., Inc. (Tokyo, Japan). Turmeric tablets were obtained from Yamada Kenkodo (Tokyo, Japan) and milk (Morinaga Milk Industry Co., Ltd., Tokyo) was purchased from a local market. One turmeric tablet $(0.4 \mathrm{~g})$ contains $0.6 \mathrm{mg}$ protein, $50 \mathrm{mg}$ lipids, $250 \mathrm{mg}$ carbohydrates, and $80 \mathrm{mg}$ curcumin according to the manufacturer's specifications.

\section{Study population and design}

The study was open, randomized and crossover designed. Eligible participants were 7 healthy male and female volunteers between the ages of 20 and 25 years $(23 \pm 1$, mean \pm SEM). Seven participants, consisting of 4 males and 3 females, received a single oral dose of 6 turmeric tablets with $200 \mathrm{ml}$ of milk or only milk as control within one minute and then crossovered after one-week washout. The study was carried out with approval from the Committee on Medical Ethics of International Research Center for Traditional Medicine prior to starting of the study, and Informed Consent was obtained from the participants in accord with Helsinki Declaration and following careful explanation of all study conditions and procedures. The study was conducted at the International Research Center for Traditional Medicine from June to August in 2005. The study protocol was also approved by Research Ethics Committee of Siebold University of Nagasaki.

\section{Blood sampling}

Subjects, who had not consumed any turmeric-contained foods like curry to their knowledge within the previous 3 days and also had fasted since last 9:00 PM, were examined one by one at each fixed time every 2 min from 9:00 AM after a 40-min seated rest through discharging urine, in a quiet, air-conditioned room with temperature maintained at $22-24^{\circ} \mathrm{C}$. The study drugs or control were ingested in $30 \mathrm{~min}$ after pre-dosing blood samples were collected. Blood samples were collected $30 \mathrm{~min}, 1 \mathrm{~h}, 2 \mathrm{~h}, 4 \mathrm{~h}, 8 \mathrm{~h}$, and $24 \mathrm{~h}$ after administration. During the examination, all subjects had the same menu at lunch (PM 1:30) and dinner (PM 6:00), and afterwards any food besides water was not allowed until $24 \mathrm{~h}$-sampling. Sampling protocol was designed to minimize oxidation after collection. Venous blood $(10 \mathrm{ml})$ was collected from subjects in heparinized tubes, maintained at room temperature throughout preparation. For plasma isolation, blood was centrifuged at 3500 rpm for $10 \mathrm{~min}$ at $10^{\circ} \mathrm{C}$. Plasma was removed, aliquoted and stored at $-80^{\circ} \mathrm{C}$ until analysis.

\section{Preparation of plasma extracts}

After thawed at room temperature, an aliquot $(0.5 \mathrm{ml})$ of plasma sample was transferred into a test tube containing equi-volume $(0.5 \mathrm{ml})$ of ethanol and vortexed vigorously. After removal of proteins by centrifugation at $2700 \mathrm{rpm}$ for $10 \mathrm{~min}, 25 \mu \mathrm{l}$ of $2 \mathrm{M} \mathrm{NaOH}$ and $2 \mathrm{ml}$ of $\mathrm{n}$-hexane were added to the resultant clear supernatants and stirred again. After separation of the upper organic phase from the lower water phase by centrifugation (2700 rpm, $10 \mathrm{~min})$, the upper phase was saved as a crude lipid fraction, which was stored at $-20^{\circ} \mathrm{C}$ until use. After neutralized with $2 \mathrm{M} \mathrm{HCl}(50 \mu \mathrm{l})$, the lower aqueous phase was added to n-hexane $(2 \mathrm{ml})$ and vortexed strongly. After centrifugation, the upper organic phase was collected and evaporated to dryness. The residues were dissolved in $0.3 \mathrm{ml}$ of ethanol and used as an acidic lipid fraction for GGA determination. The recovery of added standard GGA throughout these processes was in a range of $60-70 \%$.

\section{LC/MS analysis of the plasma extract}

HPLC was performed by using a Waters 2690 separation module (Waters, Milford, MA) equipped with a Cosmosil reverse-phase 5C18-PAQ column (4.6 mm $\times 250 \mathrm{~mm}$ Nacalai Tesque, Tokyo, Japan). The mobile phase consisted of $88 \%$ $(\mathrm{v} / \mathrm{v})$ methanol aqueous solution at a flow rate of $0.3 \mathrm{ml} / \mathrm{min}$. $1 \mu \mathrm{l}$ of each sample was injected into the column by the automatic injector. Inline MS was performed by using a Waters ZMD 2000 with an electrospray interface in the negative or positive ion mode, specifically with a capillary voltage of $3 \mathrm{kV}$ and a cone voltage of $30 \mathrm{~V}$. Dehydrated nitrogen was used for both cone gas $(56 \mathrm{l} / \mathrm{h})$ and desolvation gas $(496 \mathrm{l} / \mathrm{h})$, with the source and desolvation temperatures being held at $130^{\circ} \mathrm{C}$ and $350^{\circ} \mathrm{C}$, respectively. The data acquisition program (MassLynx NT, version 3.5, Waters) was set to scan in the selected ion mode at $\mathrm{m} / \mathrm{z} 303.4$ negative ions. 


\section{Methylation with diazomethane}

The methylation with diazomethane was conducted with authentic GGA and plasma extracts in the same procedure. An aliquot of samples was dried under nitrogen, and the residues were treated with diazomethane, Diazomethane was released from $130 \mathrm{mg}$ of MNNG by addition of $0.5 \mathrm{ml}$ water and $0.6 \mathrm{ml} 5 \mathrm{M} \mathrm{NaOH}$, and the generated gas was trapped in $3.0 \mathrm{ml}$ of ice-cold diethyl ether in a diazomethane generator (millimole size, GL Sciences, Tokyo, Japan). A sufficient amount $(0.5 \mathrm{ml})$ of the diazomethane-ether solution was added to the dry residues, and the mixture was left for $30 \mathrm{~min}$ at room temperature. After the methylation reaction, the solvent was redissolved in $250 \mu \mathrm{l}$ of ethanol. The methylated plasma extracts were analyzed on LC/MS in comparison with the methylated GGA standard.

Quantitative measurement of GGA by selected ion recording

The standard curve for quantification of GGA was made from mass peak areas of ion plots in the selected ion recording (SIR) at $\mathrm{m} / \mathrm{z} 303.4$ of the negative ion using MassLynx NT software, version 3.5. GGA standard solution $(1.0 \mu \mathrm{l})$ was injected into the reverse-phase column ranging in amounts from $0.1 \mathrm{ng}$ to $1 \mathrm{ng}$, with the regression values for $R^{2}=0.9996$ and detected by LC/MS. The concentrations of GGA in plasma extracts were calculated from the mean mass area by SIR-LC/MS chromatogram with triplicate injection based upon the standard curve.

\section{Determination of GGA in turmeric tablets and cow milk}

Turmeric tablets were extracted with ethanol at $4^{\circ} \mathrm{C}$ overnight. After evaporation to dryness, the residues were dissolved in ethanol. An aliquot $(0.5 \mathrm{ml})$ of milk was transferred into a test tube containing equi-volume $(0.5 \mathrm{ml})$ of ethanol and vortexed vigorously. After removal of proteins by centrifugation at $2700 \mathrm{rpm}$ for $10 \mathrm{~min}, 25 \mu \mathrm{l}$ of $2 \mathrm{M}$ $\mathrm{NaOH}$ and $2 \mathrm{ml}$ of n-hexane were added to the resultant clear supernatants and stirred again. After removing the upper organic phase from the lower water phase by centrifugation $(2700 \mathrm{rpm}, 10 \mathrm{~min})$ and neutralize with $2 \mathrm{M} \mathrm{HCl}$ $(50 \mu \mathrm{l})$, the lower aqueous phase was added to n-hexane ( $2 \mathrm{ml}$ ) and vortexed strongly. After centrifugation, the upper organic phase was evaporated to dryness and dissolved in ethanol and used for GGA determination.

The ehanolic solutions of turmeric tablets and cow milk were analyzed by LC/MS same procedure as plasma GGA.

\section{Results}

GGA in commercially available turmeric tablets and cow milk

At the initial place of the present study, we measured GGA contents in commercially available turmeric tablets and cow milk. As shown in Fig. 1A, authentic GGA and
ARA were eluted at around $18 \mathrm{~min}$ and $14 \mathrm{~min}$, respectively, when mass ions of $\mathrm{m} / \mathrm{z}(-)=303.4$ were traced on LC/MS. Fig. 1B clearly describes that cow milk contained free ARA, a concentration of which was calculated to be $0.3 \mu \mathrm{g} / \mathrm{ml}$ from its peak area. On the other hand, cow milk obtained from a supermarket contained no detectable amount of GGA in our assay system (Fig. 1C) so that cow milk was used as negative control food for the present human study.

In contrast, a major peak of $\mathrm{m} / \mathrm{z}(-)=303.4$ was eluted at around $5 \mathrm{~min}$ with the extract of the turmeric tablet (Fig. 1D), indicating that no free ARA nor GGA were major constituents of the turmeric tablet extract. However, a discrete peak became visible at around RT of authentic GGA when the same chromatogram was expanded by 5-fold in y-axis (Fig. 1E). Furthermore, this minor peak was precisely cochromatographed with authentic GGA added internally (Fig. 1F), indicating that a minor peak of $\mathrm{m} / \mathrm{z}(-)=$ 303.4 represents natural GGA in the turmeric tablets. The content of GGA in the turmeric tablets was calculated to be $0.28 \pm 0.22 \mu \mathrm{g} /$ tablet, consistent with our previous study [9] in which GGA contents were found highest in turmeric.

\section{Detection of endogenous GGA in human plasma}

Fig. 2 shows the elution profiles of $\mathrm{m} / \mathrm{z}(-) 303.4$ in SIR mode with the acidic lipid extracts of human plasmas before and after oral administration of the turmeric tablets or cow milk. In a routine analysis of $\mathrm{m} / \mathrm{z} 303.4 \mathrm{SIR}$, a major single peak of ARA was easily confirmed, but other distinct peak was barely detectable in human plasma before the turmeric intake (Fig. 2A). However, on the expanded scale of y-axis in the same LC/MS chromatogram, a tiny but discrete peak of $\mathrm{m} / \mathrm{z}(-)=303.4$ appeared at an RT of $18 \mathrm{~min}$ right after the descending slope of ARA peak (Fig. 2D), corresponding to standard GGA. Furthermore, the area of this peak was apparently increased in $2 \mathrm{~h}$ after the turmeric intake (Fig. 2E), although it is difficult to recognize a peak of GGA in ARA-scale chromatogram (Fig. 2B). These data suggest that the preformed GGA in the turmeric tablet may be rapidly absorbed through intestinal wall and appeared in peripheral circulation as its intact form which might be bound to albumin.

In a sharp contrast, no increase in the peak area of GGA was detected after the cow milk intake, as shown in Fig. 2F. There was also no increase in the peak area of ARA in $2 \mathrm{~h}$ after the intake of cow milk (Fig. 2C).

\section{Changes in plasma concentrations of GGA after intake of turmeric tablets}

Because the basal levels $(7.5-15.5 \mathrm{ng} / \mathrm{ml})$ of plasma GGA were variable among the individual volunteers, timedependent changes in plasma GGA and ARA concentrations after the intake of the turmeric tablet (Fig. 3A) or cow milk (Fig. 3B) were plotted as \% of their each basal level. Plasma 

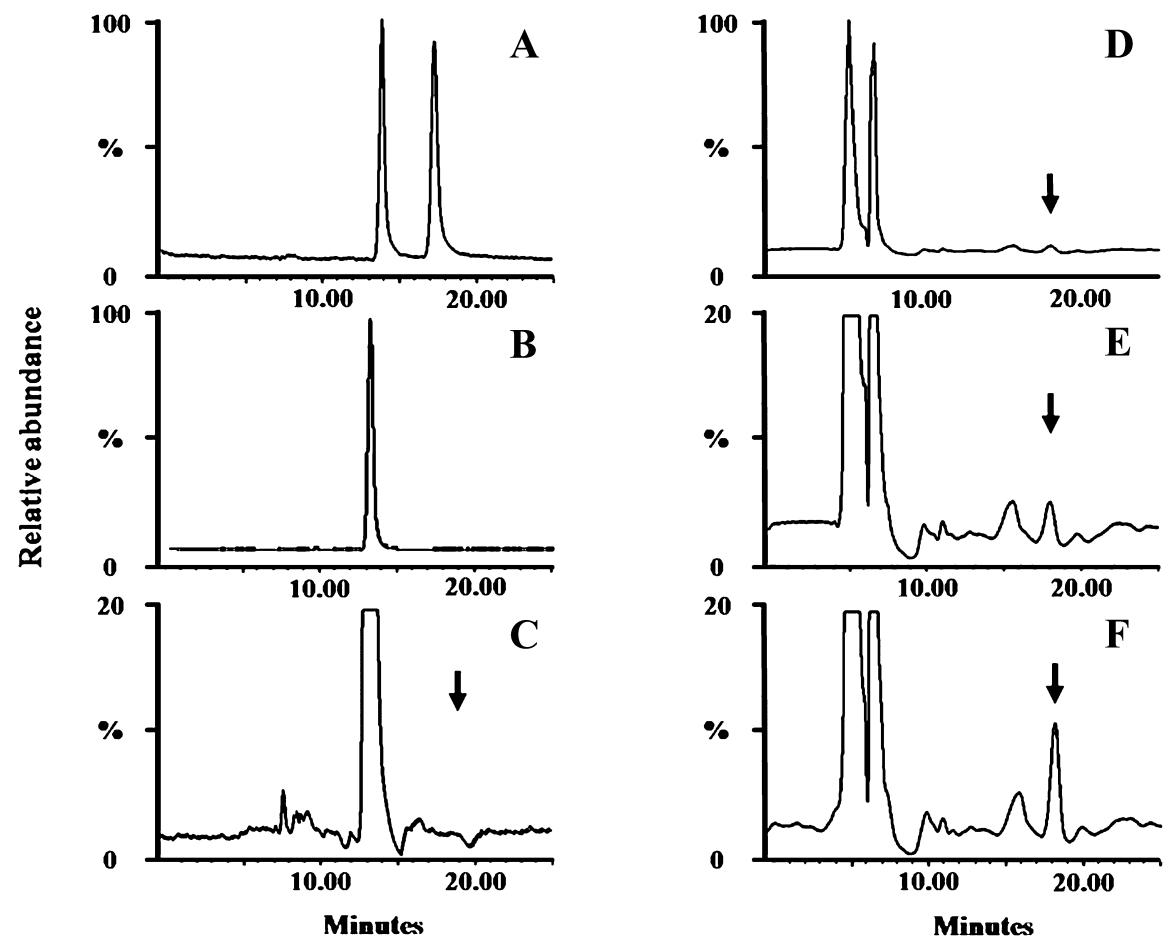

Fig. 1. LC/MS elution profiles of selected ion chromatograph (negative ions at $\mathrm{m} / \mathrm{z} 303.4$ ) of turmeric tablets and cow milk extracts. A: Authentic GGA and ARA were eluted at the RT of $17.29 \mathrm{~min}$ and $13.78 \mathrm{~min}$, respectively. B: A major component in the acidic lipid fraction of the commercial cow milk was eluted at the same RT as that of authentic ARA. C: The 5-fold expanded version (y-axis) of the chromatogram in panel B is shown to demonstrate no detectable GGA in the cow milk extracts. D: The acidic lipid fraction of the turmeric tablet was eluted to show no detectable ARA. E: The 5-fold expanded version (y-axis) of the chromatogram in panel D is to demonstrate a distinct peak of GGA. F: The cochromatogram of standard GGA with the acidic lipid fraction of the turmeric tablet is shown at the same scale as panel E. The down-pointing black arrows indicate the elution position of authentic GGA.

GGA concentrations were significantly increased in $2 \mathrm{~h}$ after the intake of the turmeric tablets and were maintained at higher level until $4 \mathrm{~h}$, and returned to the basal levels at $8 \mathrm{~h}$ (Fig. 3A). Plasma concentrations of free ARA in the same plasmas, however, stayed unchanged after the turmeric intake.

On the contrary, plasma GGA and ARA concentrations were in the range of the basal levels with a slight deviation after the milk intake (Fig. 3B).

\section{Discussion}

In the present study, we were able to detect endogenous GGA in human plasma of healthy volunteers for the first time. In addition, we could also demonstrate that plasma GGA concentration was increased after oral administration of a commercially available turmeric tablet.

A placebo-controlled double-blinded randomized clinical trial revealed the efficacy of 1-yr administration of 4,5dehydroGGA to the post-operative patients in 5-yr prevention of second primary hepatoma [8]. We have also shown that other GGA derivatives including 14,15-dihydroGGA, 2,3-dihydroGGA and GGA itself were the same potent inducers of cell death in hepatoma cells as 4,5-dehydroGGA [9]. And most importantly, GGA has recently been found as a natural constituent of several medicinal herbs including turmeric [9]. The present study additionally confirmed that natural GGA was in the extract of a commercially available turmeric tablet, which is consistent with this previous finding. Therefore, we used a commercial turmeric tablet as a GGA source in the present human study. At the initial point of the present study, we had checked the contents of GGA in several turmeric tablets and capsules made by 4 companies. The tablet used in the present study was the highest in GGA contents and no GGA was detected in tablets from other 2 companies.

We were able to detect the basal levels of GGA in human plasma for the first time. Although we are unaware of where plasma free GGA was from and what it was derived from, we have found geranylgeraniol $(\mathrm{GGOH})$ and geranylgeranyl pyrophosphate (GGPP) in ordinary foods like polished rice, a principal food for Japanese (manuscript in preparation). 

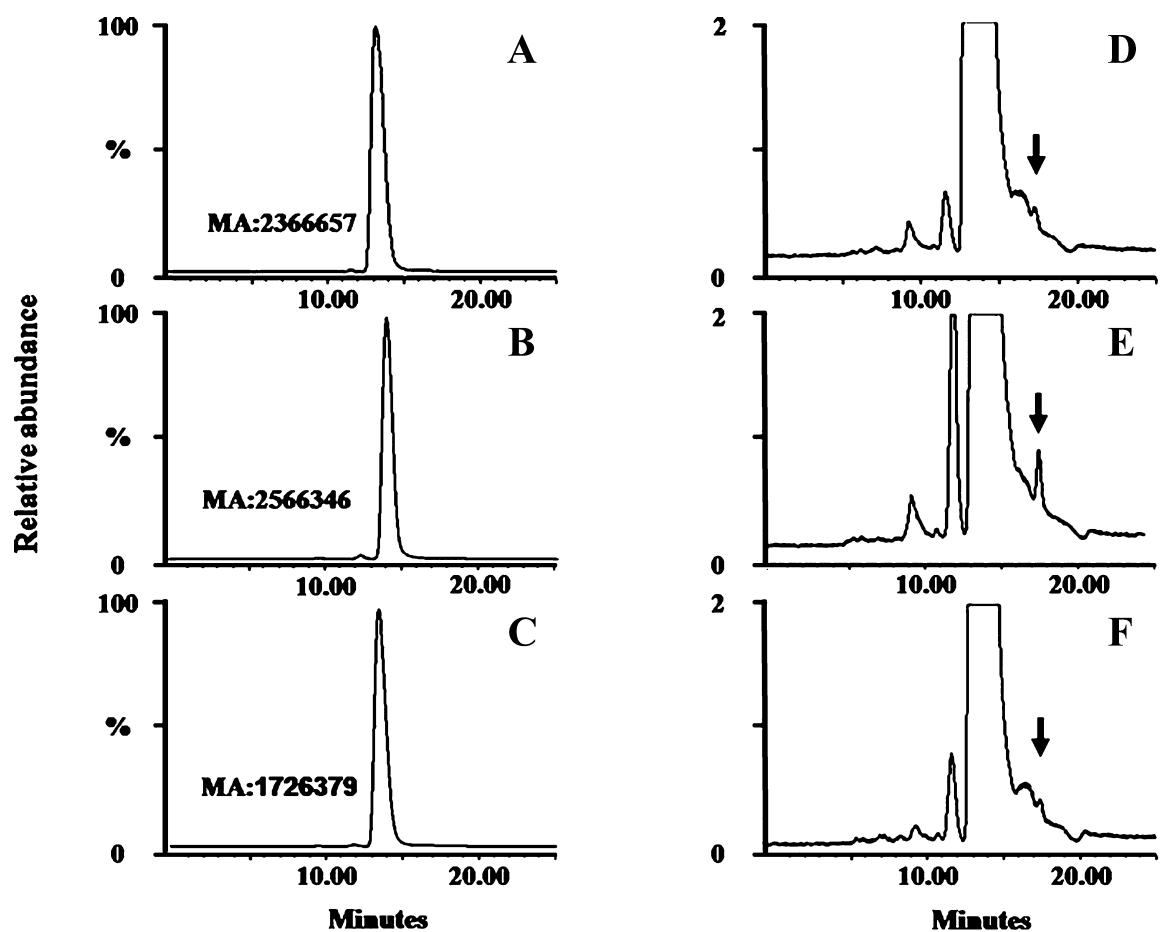

Fig. 2. LC/MS analysis of plasma extracts before and after oral administration of turmeric tablets or milk. LC/MS analysis was conducted as described in the "Materials and Methods" section. A and D: Elution profiles of m/z(-) 303.4 in the acidic lipid fraction from plasma taken before oral administration of the turmeric tablet. The 50-fold expanded version in y-axis of the chromatogram in panel $\mathrm{A}$ is depicted in panel D to demonstrate circulating GGA. B and E: Elution profiles of m/z(-) 303.4 in the acidic lipid fraction from plasma taken $2 \mathrm{~h}$ after oral administration of the turmeric tablet. The 50-fold expanded version in $\mathrm{y}$-axis of the chromatogram in panel $\mathrm{B}$ is depicted in panel $\mathrm{E}$ to demonstrate the increased amount of circulating GGA. C and F: Elution profiles of $\mathrm{m} / \mathrm{z}(-) 303.4$ in the acidic lipid fraction from plasma taken $2 \mathrm{~h}$ after oral administration of the cow milk. The 50 -fold expanded version in $\mathrm{y}$-axis of the chromatogram in panel $\mathrm{C}$ is depicted in panel $\mathrm{F}$ to demonstrate no increase in amount of circulating GGA. MA, mass area.
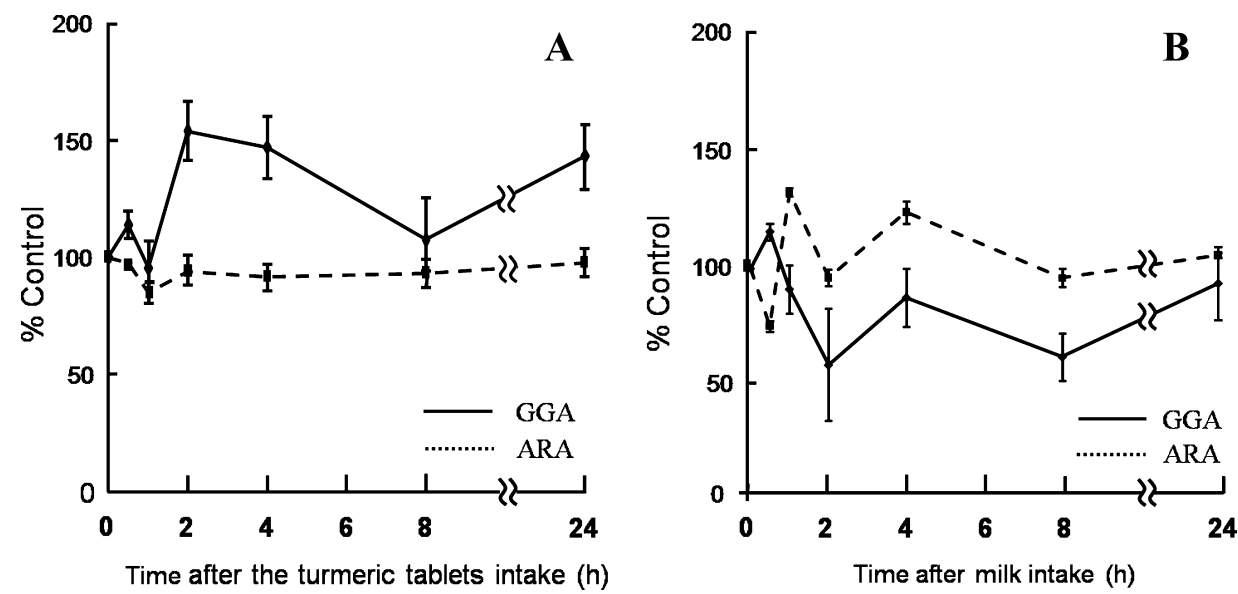

Fig. 3. Changes in the concentrations of plasma GGA and ARA after oral administration of the turmeric tablets or cow milk. LC/MS analysis of plasma GGA and ARA was conducted as described in the "Materials and Methods" section. Time-dependent changes in plasma GGA and ARA concentrations after the intake of the turmeric tablets (A) or cow milk (B) were plotted as \% of their each basal level of circulating GGA in individuals. Each symbol represents the mean $\pm \operatorname{SE}(n=4)$. 
Therefore, one can easily speculate that GGOH and GGPP in foods daily ingested may be oxidatively metabolized to GGA in our body. In fact, bovine intestinal alkaline phosphatase catalyzed dephosphorylation of GGPP to GGOH and an in vitro incubation of GGOH with NAD(P) in rodent liver homogenates produced GGA by stoichiometry (manuscript in preparation). In this context, the "endogenous" GGA in human plasma, which we found by LC/MS technique, might be derived from natural food resources. This may be a reason why the basal levels of GGA varied among the individuals who are usually eating GGA-rich or GGA-poor foods. The same thing is true with phytanic acid, perhydroGGA or fully-saturated GGA. The elevated level of phytanic acid in plasma is used for diagnosis of peroxisomal disorders [10] including Zellweger syndrome [11]. Phytanic acid is well known to be derived from phytol, a breakdown product of chlorophyll. Phytol is not produced in the human gut, but micro-organisms present in the gastrointestinal system of ruminants can break down chlorophyll to release phytol, which is then converted into phytanic acid in our body.

The average basal level of plasma free GGA was around $0.05 \mu \mathrm{M}$ concentration, which is in a range of one-hundredth of the concentration $(5 \mu \mathrm{M})$ inducing cell-death in human hepatoma-derived HuH-7 cells in vitro [12]. Although we never tried repeated administration of turmeric tablets in the present study, a single administration of the tablet made a slight increase of plasma GGA up to $0.07 \mu \mathrm{M}$, which concentration is also far below the toxic concentration against hepatoma cells. It may be worthwhile to mention that the ingested GGA may be selectively taken up and accumulated in tumor cells, because tumor cells show the enhanced expression of CRABP, to which GGA may be bound [3]. Meanwhile, we must also describe an apparent rebounded increase of plasma GGA concentrations at $24 \mathrm{~h}$ after tablet intake (Fig. 3A), suggesting that the upregulation of plasma GGA concentrations may not be merely due to intestinal absorption of exogenous GGA, but also due to changes of GGA metabolism after turmeric intake.

In the present study, we tested bioavailability of preformed GGA ingredient in a turmeric tablet which is commercially available in a regular drug store. As a result, a postprandial increase in plasma free GGA concentration was observed at $2 \mathrm{~h}$ after the turmeric intake. Although the time of peak plasma fatty acid concentration generally occurs 2 to $6 \mathrm{~h}$ following dose in normal healthy women [13], the time at which maximum concentration of coenzyme Q10 (CoQ10) occurs in the plasma after supplementation with CoQ10 soft gel capsule is $6 \mathrm{~h}$ or after [14, 15]. In this context, GGA may be classified into fatty acids rather than terpenoids, in terms of molecular mechanism of intestinal absorption.

We measured only free form of GGA in plasma, but
GGA can be esterified with glycerol, because GGA shares biological and chemical characteristics of branched chainand polyunsaturated fatty acids. Therefore, we are now going to establish an assay system for the putative esterified forms of GGA in plasma. Furthermore, one should be cautious about other water-soluble metabolites of GGA such as its glucronides and sulfides, because plaunotol (18-hydroxygeranylgeraniol) and 18-hydroxyGGA were reported to be conjugated with $\beta$-glucronic acid in rats and dogs [16]. We also know that dose-response type experiment of clinical trial is certainly required to establish the bioavailability of GGA in foods for the future experiment.

In conclusion, the results of the present study show that LC/MS analysis detected GGA in human plasma and the concentration of circulating GGA was increased after oral administration of a turmeric tablet, which contained preformed GGA.

\section{References}

[1] Muto, Y., Moriwaki, H., Ninomiya, M., Adachi, S., Saito, A., Takasaki, K.T., Tanaka, T., Tsurumi, K., Okuno, M., Tomita, E., Nakamura, T., and Kojima, T.: Prevention of second primary tumors by an acyclic retinoid, polyprenoic acid, in patients with hepatocellular carcinoma. Hepatoma Prevention Study Group. N. Engl. J. Med., 334, 1561-1567, 1996.

[2] Muto, Y., Moriwaki, H., and Saito, A.: Prevention of second primary tumors by an acyclic retinoid in patients with hepatocellular carcinoma. N. Engl. J. Med., 340, 1046-1047, 1999.

[3] Muto, Y., Moriwaki, H., and Omori, M.: In vitro binding affinity of novel synthetic polyprenoids (polyprenoic acids) to cellular retinoid-binding proteins. Jpn. J. Cancer Res., 72, 974-977, 1981.

[4] Araki, H., Shidoji, Y., Yamada, Y., Moriwaki, H., and Muto, Y.: Retinoid agonist activities of synthetic geranylgeranoic acid derivatives. Biochem. Biophys. Res. Commun., 209, 6672, 1995.

[5] Yamada, Y., Shidoji, Y., Fukotomi, Y., Ishikawa, T., Laneko, T., Nakagama, H., Imawari, M., Moriwaki, H., and Muto, Y.: Positive and negative regulations of albumin gene expression by retinoids in human hepatoma cell lines. Mol. Carcinog., 10, 151-158, 1994.

[6] Muto, Y. and Moriwaki, H.: Antitumor activity of vitamin A and its derivatives. J. Nat. Cancer Inst., 73, 1389-1393, 1984.

[7] Muto, Y. and Moriwaki, H.: Acylic retinoids and cancer chemoprevention. Pure Appl. Chem., 63, 157-160, 1991.

[8] Ong, T.P., Heidor, R., de Conti, A., Dagli, M.L., and Moreno, F.S.: Farnesol and geraniol chemopreventive activities during the initial phases of hepatocarcinogenesis involve similar actions on cell proliferation and DNA damage, but distinct actions on apoptosis, plasma cholesterol and HMG-CoA reductase. Carcinogenesis, 27, 1194-1203, 2006.

[9] Shidoji, Y. and Ogawa, H.: Natural occurrence of cancer- 
preventive geranylgeranoic acid in medicinal herbs. J. Lipid Res., 45, 1092-1103, 2004.

[10] Al-Dirbashi, O., Santa, T., Rashed, M., Al-Hassnan, Z., Shimozawa, N., Chedrawi, A., Jacob, M., and Al-Mokhadab, M.: Rapid UPLC-MS/MS method for routine analysis of plasma pristanic, phytanic, and very long chain fatty acid markers of peroxisomal disorders. J. Lipid Res., 49, 18551862, 2008.

[11] Rosewich, H., Gartner, J., and Krause, C.: Rational diagnostic strategy for Zellweger syndrome spectrum patients. Eur. J. Hum. Genet., 7, 741-748, 2009.

[12] Nakamura, N., Shidoji, Y., Yamada, Y., Hatakeyama, H., Moriwaki, H., and Muto, Y.: Induction of apoptosis by acyclic retinoid in the human hepatoma-derived cell line, HuH-
7. Biochem. Biophys. Res. Commun., 207, 382-388, 1995.

[13] Jones, A., Stolinski, M., Smith, R., Murphy, J., and Wootton, S.: Effect of fatty acid chain length and saturation on the gastrointestinal handling and metabolic disposal of dietary fatty acids in women. Br. J. Nutr., 81, 37-43, 1999.

[14] Miles, M.: The uptake and distribution of coenzyme Q(10). Mitochondrion, 7S, S72-S77, 2007.

[15] Evans, M., Baisley, J., Barss, S., and Guthrie, N.: A randomized, double-blind trial on the bioavailability of two CoQ10 formulations. J. Func. Foods, 1, 65-73, 2009.

[16] Ikeda, T., Kawai, K., Horiguchi, M., and Komai, T.: A novel conjugation reaction. Formation of doubly conjugated antiulcer drug CS-684, plaunotol, with fatty acid and glucronic acid. Drug Metab. Pharmacokinet., 1, 226-227, 1986. 